\title{
Space matters
}

\section{Peter Nick}

Published online: 23 February 2012

(C) Springer-Verlag 2012

As the other biological disciplines, cell biology has transcended its traditional borders and merged with other fields of biology. At first sight, this was accompanied by a certain loss of profile - what is cell biology actually doing these days? The answer to this question is much harder to formulate as compared to a few decades ago. In a period, where the unifying principles of molecular biology have been solidly rooted even in the perception of undergraduate students, the question seems justified. When the motivation of molecular biology might be described as identification of the molecules that fulfill a certain biological function, cell biology has always been driven by the question, where things happen. And this has remained valid till nowadays. In other words, cell biology asks for the organisation of biology in space. This includes, of course, the question where given molecules are located, but also addresses the spatial organisation of biological events beyond molecules. The last 20 years were mainly shaped by breakthrough technologies of molecular biology, often linked to highthroughput "-omics" approaches. The renewed interest in epigenetic phenomena has reconfirmed the importance of spatial organisation, for instance, when gene activity is linked with the spatial organisation of chromatin. It is to be expected that the postgenomic era will see a rising impact of cell biology, supported by the tremendous advances of live cell microscopy. The current issue assembles several contributions that highlight the importance of space and geometry:

\section{Handling Editor: Peter Nick}

P. Nick $(\bowtie)$

University of Karlsruhe,

Karlsruhe, Germany

e-mail: peter.nick@bio.uka.de
Geometry of cell division The review by Müller (2012) in the current issue investigates the geometrical rules that govern the selection of cell division planes in plant cells and thus gives an excellent example for a genuinely cell biological approach. From the comparison with other organisms, general biophysical and mathematical laws can be deduced and transferred to plant cells. It seems that microtubules are used as tools to perceive geometry and transduce it into a highly dynamic and nevertheless robust pattern that subsequently can organise the molecular players of mitosis and cell division in time and space. This sensory function of microtubules is linked to biophysical laws rather than to molecules and is of tremendous importance for application (for a recent review see Nick 2012).

Spatial organisation of seed storage proteins The practical relevance of space is even more evident in the field of seed storage proteins that are assigned to specific compartments in the maturating seed. The understanding of this subcellular compartmentalisation is not only relevant for efficient processing of seed crops, but progressively for the production of recombinant proteins in seeds. The review by Ibl and Stoger (2012) in the current issue surveys our current knowledge on the assignment of the proteins produced in the endoplasmic reticulum towards the dynamic protein storage vacuoles and other protein bodies. Which signals and mechanisms ensure that a given protein is targeted to the correct compartment? How are aggregation and overloading of the transport machinery avoided? How is this complex cellular activity integrated into development? How is this machinery linked to the genesis of vacuoles that specifically differs from lysosomal genesis in yeast and animal cells (see Robinson and Hinz 1997). The spatial organisation of seed storage proteins represents an impressive logistic challenge that has to be handled and is extremely relevant for the 
success of any biotechnological approach towards plant biomass.

Autophagy - a process of decompartmentalisation Compartments, such as seed storage organelles, are not only dynamically interchanged, but can also be disintegrated to mobilise nutrients or to remove damaged organelles. This so called autophagy, reviewed by Avin-Wittenberg et al. (2012) in the current issue, has been known for a longer time from fungal and mammalian cells, represents an emerging topic also in the plant field. Again, some of the conserved components are still missing in plants, whereas others are encoded by enlarged gene families. These plant-specific differences might be linked to plant-specific developmental processes such as the mobilisation of resources in the germinating seed.

Spatial organisation of stress signaling Reactive oxygen species play a central role in stress signaling and adaptation in most organisms. Their role is twofold - on the one hand, they can cause cellular damage by attacking proteins, lipids and even DNA. On the other hand, they are central signals that trigger and control in a very specific manner adaptive responses against abiotic and biotic stress. How can such simple molecules that virtually interact with almost everything that crosses their way be specific signals? The answer is again: it is space that matters. The subcellular distribution of oxidative burst provides the specificity that is required for successful adaptation. In an electron-microscopical study, Yamane et al. (2012) in the current issue follow the subcellular distribution of hydrogen peroxide in response to salt stress and provide high-resolution information on thylakoid structure in the important staple crop rice. They can show the accumulation of hydrogen peroxide around thylakoids depending on photosynthetic activity, and they can rescue a normal configuration of thylakoids by scavenging reactive oxygen species by ascorbate showing that these oxygen species are responsible for the ultrastructural response.

Plant exocytosis carriers Exocytosis allows excretion of molecules without the need for membrane passage. In cells endowed with polar growth, this excretion is extremely localised and controlled by a complex, phosphoinositidedependent machinery (for a recent review see Ischebeck et al. 2010). However, also in plant cells with diffuse growth, exocytotic activity has to be organised in space. In animal cells, so-called SCAMPs (for secretory carrier membrane proteins) are important mediators. In their review, Law et al.
(2012) in the current issue summarise the still scarce knowledge about plant homologues of this protein group. From the comparison with their animal counterparts, they can identify important similarities, but also, specific differences based on work with Arabidopsis thaliana, they deduce first ideas and concepts for the biological function of plant SCAMPs.

Spatiotemporal organisation of alkaloid metabolism Alkaloids are of tremendous medical interest. However, attempts to produce them by molecular farming in cell cultures have failed so far, despite considerable effort. The reason might be found in the incredibly complex spatiotemporal organisation of metabolism. Using the example of the precious Madagascar Periwinkle alkaloid vinblastine Verma et al. (2012) in the current issue summarise the state of the art and discuss how at least four cell types (epidermis, internal phloëm associated parenchyma, laticifers and idioblasts) and five intra-cellular compartments (chloroplast, vacuole, nucleus, endoplasmic reticulum and cytosol) have to cooperate to produce a total of more than 130 bioactive terpenoid indole alkaloids and provide an important base on new strategies to get access to these compounds that have great pharmacological value, are only scarcely produced in planta, cannot be synthetically substituted and therefore are traded at exorbitant market costs.

\section{References}

Avin-Wittenberg T, Honig A, Galili G (2012) Variations on a theme: plant autophagy in comparison to yeast and mammalians. Protoplasma: current issue

Ibl V, Stoger E (2012) The formation, function and fate of protein storage compartments in seeds. Protoplasma: current issue

Ischebeck T, Seiler S, Heilmann I (2010) At the poles across kingdoms: phosphoinositides and polar tip growth. Protoplasma 240:13-31

Law AHY, Chow CM, Jiang L (2012) Secretory carrier membrane proteins. Protoplasma: current issue

Müller S (2012) Universal rules for division plane selection in plants. Protoplasma doi:10.1007/s00709-011-0289-y

Nick P (2012) Microtubules and the tax payer. Protoplasma doi:10.1007/s00709-011-0339-5

Robinson DG, Hinz G (1997) Vacuole biogenesis and protein transport to the plant vacuole: a comparison with the yeast vacuole and mammalian lysosome. Protoplasma 197:1-25

Verma P, Mathur AK, Srivastava A, Mathur A (2012) Emerging trends in research on spatial and temporal organization of terpenoid indole alkaloids pathway in Catharanthus roseus: a literature up-date. Protoplasma: current issue

Yamane K, Taniguchi M, Miyake H (2012) Salinity-induced subcellular accumulation of $\mathrm{H}_{2} \mathrm{O}_{2}$ in leaves of rice. Protoplasma: current issue 